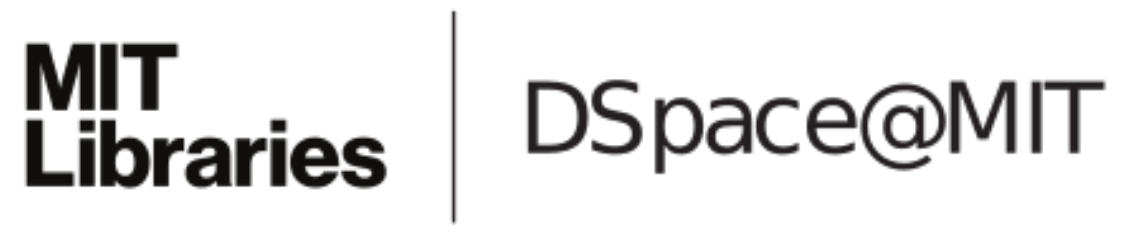

\author{
MIT Open Access Articles
}

Modeling Traveling Waves Using Mode Superposition

The MIT Faculty has made this article openly available. Please share how this access benefits you. Your story matters.

Citation: Jaiswal, Vivek, Aditi Sheshadri, and J. Kim Vandiver. "Modeling Traveling Waves Using Mode Superposition." 29th International Conference on Ocean, Offshore and Arctic Engineering: Volume 1 (2010).

As Published: http://dx.doi.org/10.1115/OMAE2010-20807

Publisher: American Society of Mechanical Engineers

Persistent URL: http://hdl.handle.net/1721.1/109293

Version: Final published version: final published article, as it appeared in a journal, conference proceedings, or other formally published context

Terms of Use: Article is made available in accordance with the publisher's policy and may be subject to US copyright law. Please refer to the publisher's site for terms of use. 


\title{
OMAE2010-प०००
}

\section{Modeling Traveling Waves using Mode Superposition}

\author{
Vivek Jaiswal \\ Granherne, Inc \\ Houston, Texas, USA
}

\author{
Aditi Sheshadri \\ Metacomp Technologies \\ Agoura Hills, California, USA
}

\author{
J. Kim Vandiver \\ Massachusetts Institute of Technology \\ Cambridge, Massachusetts, USA
}

\begin{abstract}
Analysis of the data from two Vortex-Induced Vibration (VIV) experiments conducted in the Gulf Stream on a 500foot-long, 1.43 inches diameter, flexible, tension dominated riser model revealed that the response is predominantly characterized by the presence of traveling waves. It was also observed that the location of the VIV excitation region (power-in) affects the characteristics of the response. The conventional method of modeling the excitation force as a standing wave was found inadequate to predict the location of the peak measured response accurately, especially in the cases where the excitation region is close to a boundary (the ends of the riser model).
\end{abstract}

A modified excitation force model consisting of a combination of standing and traveling wave excitation regions is demonstrated to predict the location of the peak response more accurately. This work presents the idea of modifying the VIV excitation model to include traveling wave characteristics and using mode superposition method for computing the response to this modified force. Examples of the implementation of this method are shown for the two distinct cases of the location of the power-in region - the power-in region adjacent to the boundary and the power-in region away from the boundary. Depending on the location of the power-in region, different proportions of standing and traveling wave excitations are used to yield predicted responses that match the measured response characteristics.

\section{INTRODUCTION}

Offshore marine risers and pipelines, exposed to ocean currents, are susceptible to VIV. These vibrations lead to fatigue which can limit the functional life of the offshore structures. Depleting oil and gas resources on land and in shallow water offshore have forced the oil and gas industry to explore further offshore in deeper waters. The exploration depth, which stood at $5000 \mathrm{ft}$ in the early 1980 's, has doubled since. The production depth has gone up from $1000 \mathrm{ft}$ to nearly $7000 \mathrm{ft}$ in the same time period. The increased water depth necessitates the use of risers and pipelines with high aspect ratio (length to diameter ratio).

The VIV of high aspect ratio structures is relatively less understood. These structures, due to their high aspect ratio, have a higher shedding frequency to first natural frequency ratio. Therefore, for a given current environment, high aspect ratio structures respond at higher-mode frequencies compared to low aspect ratio structures. The authors refer to modes numbers above the tenth mode as high mode numbers.

A DEEPSTAR sponsored VIV experiment campaign on a high aspect ratio riser model was conducted in the Gulf Stream. The objective of the experiment was to obtain data from a densely instrumented riser model that can 
lead to a better understanding of VIV of long flexible cylinders and its prediction.

\section{GULF STREAM EXPERIMENTS}

The Gulf Stream Experiments were conducted on the Research Vessel F. G. Walton Smith from the University of Miami using a fiber glass composite pipe, 500.4-foot-long and 1.43 inches in outer diameter. The experiment consisted of towing the pipe in the Gulf Stream. The set-up of the experiment is shown in Figure 1. A railroad wheel weighing $805 \mathrm{lbs}$ (dry weight, $725 \mathrm{lbs}$ in water), was attached to the bottom of the pipe to provide tension. Strain gauges were used to measure the VIV response of the pipe. 8 optical fibers containing 35 strain gauges each were embedded in the outer layer of the composite pipe. Each quadrant of the pipe had two fibers embedded so that in all there were 70 strain gauge measurements available. The spacing between the adjacent strain gauges in a quadrant was $7 \mathrm{ft}$. An ADCP was used to record the current velocity and direction along the length of the pipe. More details about the experiment can be found in $[1,2,5]$.

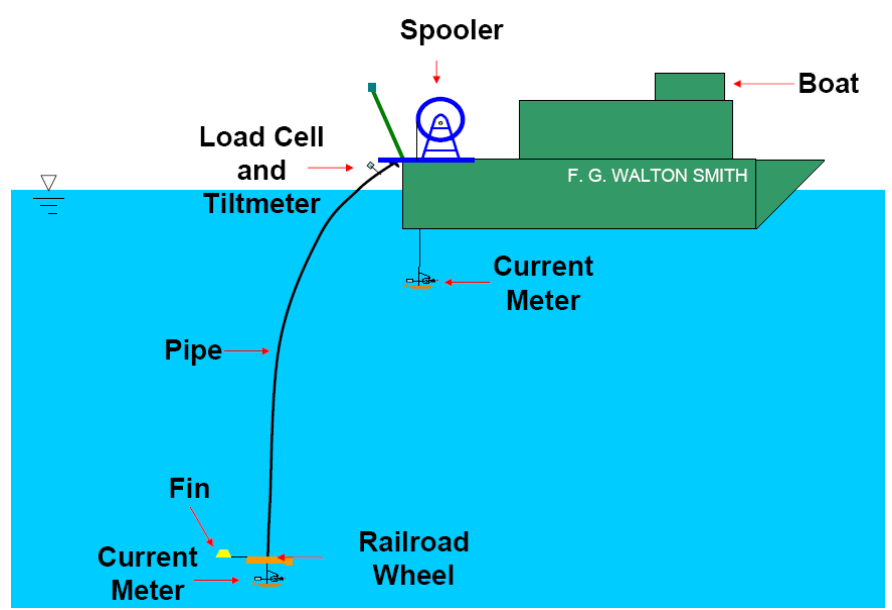

Figure 1 Set-up for the Gulf Stream Experiments.

\section{CHARACTERISTICS OF HIGH MODE NUMBER VIV RESPONSE DATA}

Analysis of the data from the Gulf Stream experiments reveals that the cross-flow response is characterized by the presence of traveling waves [1,3]. Another interesting feature of the measured response is that the maximum strain is not at the same spatial location as the maximum incident current. Figure 2 shows the current profile and the root mean square (RMS) strain from one of the test cases (20061023205557) and illustrates the above mentioned point. This unexpected response behavior is partially explained by the significant presence of odd higher harmonic $3 \mathrm{X}$ frequency (three times the primary crossflow response frequency which is denoted as $1 \mathrm{X}$ ) in the cross-flow direction [4].
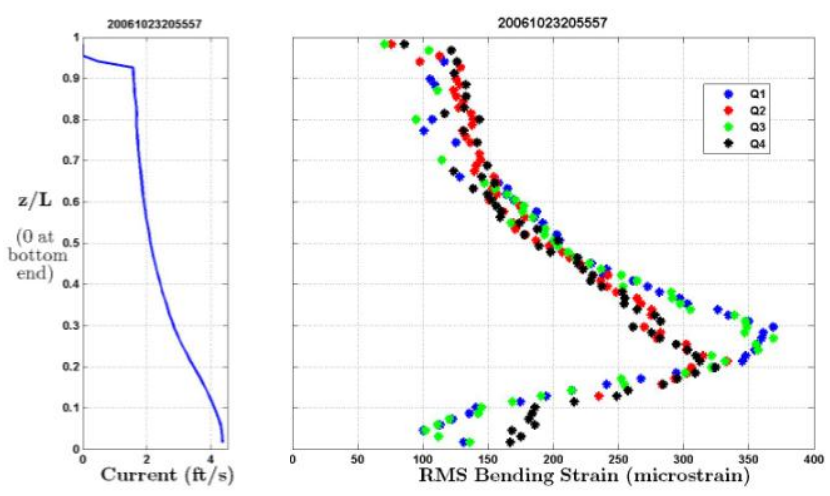

Figure 2 Current profile and RMS strain for test case 20061023205557. Strain data from all 4 quadrants of the pipe is shown.

The plot on the right in Figure 3 shows the RMS strain due to just the $1 \mathrm{X}$ response frequency for test case 20061023205557. The plot on the left shows the current profile $(\mathrm{U}(\mathrm{z}))$ and the reduced velocity $\left(\mathrm{V}_{\mathrm{r}}(\mathrm{z})\right)$ based on the $1 \mathrm{X}$ response frequency $\left(\mathrm{f}_{1 \mathrm{x}}\right)$ and pipe diameter $\mathrm{d}$ :

$$
\mathrm{V}_{\mathrm{r}}(\mathrm{z})=\mathrm{U}(\mathrm{z}) /\left(\mathrm{f}_{1 \mathrm{x}} \mathrm{d}\right)
$$

The region with reduced velocity between 5 and 7 is also marked out in the plot. This region is the expected powerin region and extends from the bottom end of the riser model to approximately $z / L=0.2$. It is evident from this figure that contrary to the conventional understanding, the maximum $1 \mathrm{X}$ cross-flow strain is not inside the power-in region but closer to the end of the power-in region.

Figure 4 shows RMS strain data due to $1 \mathrm{X}$ response frequency for another test case (20061022153003). The measured current profile and the reduced velocity (as defined in equation 1) are also shown. In this example the maximum measured current is at a location which is away from the boundary ( $\mathrm{z} / \mathrm{L}=0.28$ ). In this case, the measured response shows two peak, near $\mathrm{z} / \mathrm{L}=0.4$ and the other near $z / L=0.2$. Both these peaks are located towards the ends of the power-in region and as in the previous example; the maximum strain location does not coincide with the location of the maximum current. 

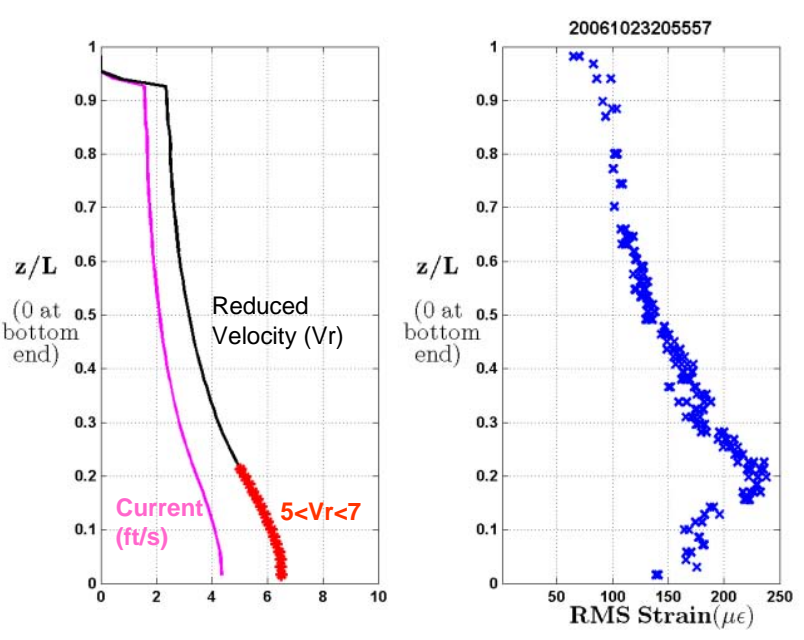

Figure 3 Current, Reduced Velocity and 1X RMS strain for test case 20061023205557.
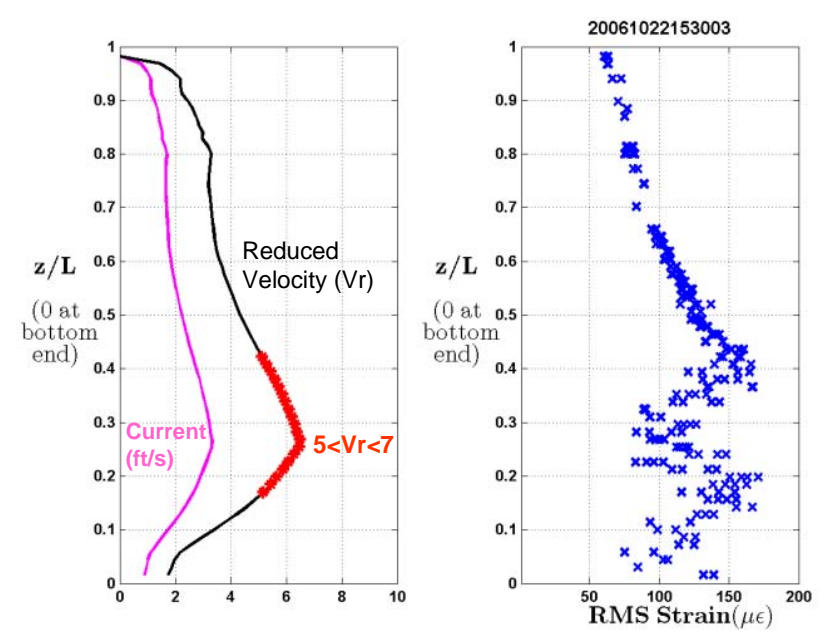

Figure 4 Current, Reduced Velocity and 1X RMS strain for test case 20061022153003 .

\section{MOTIVATION}

Response prediction techniques which can accurately predict the response due to VIV can help in deciding how to mitigate it, and also to predict the fatigue life of the structure. It is desirable that a prediction method should be able to predict, with reasonable accuracy, both the maximum response as well as the location of the maximum response.

Conventional VIV response prediction techniques assume that the magnitude of the excitation force is a function of the local response amplitude and reduced velocity and is harmonic in time. The conventional VIV excitation force $P(\mathrm{z}, \mathrm{t})$ is in the form of a standing wave and can be written as:

$$
P(z, t)=f(z) \cos (\omega t+\phi)
$$

Figure 5 shows the predicted RMS strain using the conventional standing wave excitation force and mode superposition method for the Gulf Stream test case 20061023205557. The details of the method of computing the response using the mode superposition follows in the next section. The excitation region ( $\mathrm{z} / \mathrm{L}=0$ to 0.2 ) is marked out with a black bar in the plot on the right. The excitation force is of the form shown in equation 2, with $f(z)$ being a real valued function.

$$
f(z)=\frac{1}{2} \rho U^{2}(z) d C_{L}(z)
$$

In the above equation, $U(z)$ is the incident current speed and $C_{L}(z)$ is the Lift coefficient, $\rho$ is the density of the fluid and $d$ is the diameter of the riser model. The values of $C_{L}(z)$ were obtained from the commercial VIV response prediction program Shear7.

The pipe properties and Shear7 specific parameters used for modeling the response are as follows:

\begin{tabular}{|l|l|}
\hline Inner Diameter & $0.98 \mathrm{in} .(0.0249 \mathrm{~m})$ \\
\hline Outer Diameter & $1.43 \mathrm{in} .(0.0363 \mathrm{~m})$ \\
\hline EI & $1.483 \mathrm{e} 3 \mathrm{lb} \mathrm{ft}^{2}\left(613 \mathrm{Nm}^{2}\right)$ \\
\hline EA & $7.468 \mathrm{e} 5 \mathrm{lb}(3.322 \mathrm{e} 6 \mathrm{~N})$ \\
\hline Weight in Seawater & $\begin{array}{l}0.1325 \quad \mathrm{~b} / \mathrm{ft}(0.1972 \\
\mathrm{kg} / \mathrm{m})\end{array}$ \\
\hline Weight in air & $0.511 \mathrm{lb} / \mathrm{ft}(0.760 \mathrm{~kg} / \mathrm{m})$ \\
\hline Effective mean tension & $\begin{array}{l}725 \mathrm{lb}(3225 \quad \mathrm{~N}, \text { wet } \\
\text { weight of railroad } \\
\text { wheel })\end{array}$ \\
\hline Length & $500.4 \mathrm{ft}(152.524 \mathrm{~m})$ \\
\hline Structural damping ratio & 0.003 \\
\hline Added mass coefficient & 1 \\
\hline Strouhal number & 0.16 \\
\hline Lift coefficient Table & 2 \\
\hline $\begin{array}{l}\text { Hydrodynamic damping } \\
\text { coefficients }\end{array}$ & $0.2,0.18,0.2$ \\
\hline $\begin{array}{l}\text { Reduced velocity } \\
\text { bandwidth }\end{array}$ & 0.4 \\
\hline $\begin{array}{l}\text { Power cutoff, primary } \\
\text { zone amplitude limit }\end{array}$ & $0.7,0.3$ \\
\hline
\end{tabular}

The program computes the Lift coefficient distribution corresponding to the discrete frequencies (modes) which 
have significant contribution in the response [6]. It also computes the response for each of these frequencies. In the example shown in Figure 5 and in the second example presented later in the paper, the Lift coefficient distribution for the frequency with the largest modal amplitude was selected.

It can be seen from this example that the use of the conventional VIV response prediction method, where the excitation force is modeled as a standing wave, predicts the amplitude of the peak response with reasonable accuracy but the spatial location of the peak is inaccurate. The conventional method predicts the peak response at about a quarter wavelengths away (the location of an anti-node for the mode corresponding to the response frequency) from the boundary.

Jaiswal [1] has demonstrated that the excitation force model needs to be modified to capture the spatial variation of the response as measured in the Gulf Stream test cases. It was demonstrated that in the case where the power-in region was close to a boundary, the excitation force was required to be modeled as a standing wave excitation in part of the powerin region close to the boundary and as a traveling wave in part of the power-in region away from the boundary.

Mode superposition is a widely used method for dynamic response computation of structures. It has been successfully used for VIV response prediction using the conventional standing wave excitation force model. This paper extends the application of mode superposition method for response computation to an excitation force with traveling wave characteristics. The similarities and the differences in the computational steps in mode superposition using the standing and traveling wave excitation forces are presented in the following section.

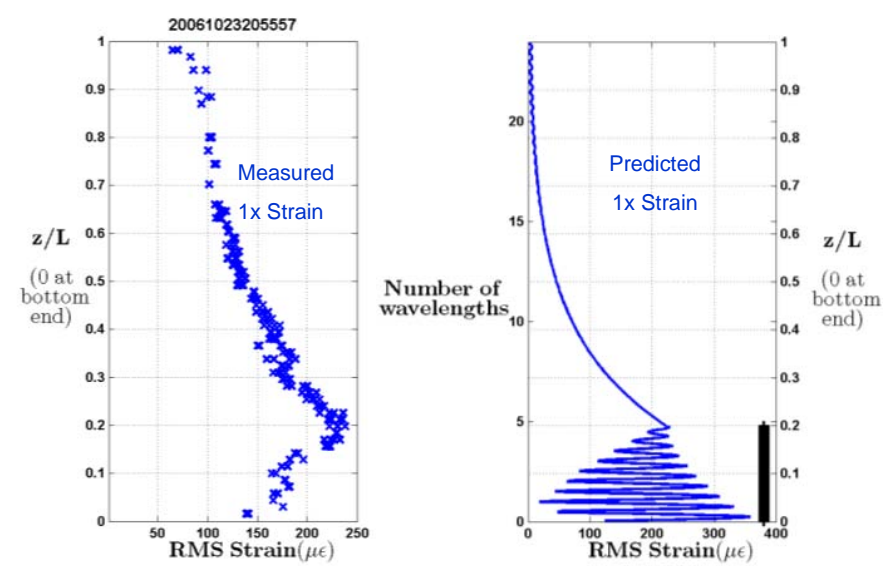

Figure 5 Measured (left) and predicted (right) RMS strain for test case 20061023025557 using the conventional standing wave excitation between $\mathrm{z} / \mathrm{L}=0$ to 0.2.

\section{MODE SUPERPOSITION METHOD FOR MODELING TRAVELING WAVE EXCITATION}

Consider the equation of motion of a tensioned string of length $\mathrm{L}$ that is excited by the distributed force $P(\mathrm{z}, \mathrm{t})$ :

$\rho \frac{\partial^{2} y}{\partial t^{2}}+r \frac{\partial y}{\partial t}-T \frac{\partial^{2} y}{\partial z^{2}}=P(z, t)$

Where $T$ is the tension in the string, $\rho$ is the mass per unit length of the string, $y(z, t)$ is the transverse displacement, $r$ represents damping (both structural and environmental, here it is assumed that the damping is independent of spatial position), and $P(z, t)$ is the excitation force along the string. The excitation force is harmonic and has a frequency of $\omega_{r}$. This force is extended from $\mathrm{z}_{\mathrm{s}}$ to $\mathrm{z}_{\mathrm{e}}$ (both $z_{\mathrm{s}}$ and $z_{\mathrm{e}}$ are within $[0, \mathrm{~L}]$ and $\mathrm{z}_{\mathrm{s}}$ is less than or at most equal to $z_{\mathrm{e}}$. If the string is in water, $\rho$ should include added mass and $T$ should be the effective tension.

The system displacement response can be written as

$$
y(z, t)=\sum_{n} Y_{n}(z) q_{n}(t)
$$

Where $Y_{n}(t)$ is the $\mathrm{n}^{\text {th }}$ mode shape of the system and $q_{n}(t)$ is the $\mathrm{n}^{\text {th }}$ modal displacement.

Substituting this relation into the governing equation of the string and following the standard procedure of modal analysis, leads to

$$
M_{n} \ddot{q}_{n}(t)+R_{n} \dot{q}_{n}(t)+K_{n} q_{n}(t)=P_{n}(t)
$$

Where $M_{n}$ is the modal mass and is given by

$M_{n}=\int_{0}^{L} \rho Y_{n}^{2}(z) d z ;$

$R_{n}$ is the modal damping and is given by

$R_{n}=\int_{0}^{L} Y_{n}^{2}(z) r d z$

(it is assumed that damping is such that the governing equation can be decoupled);

$K_{n}$ is the modal stiffness and is given by

$K_{n}=-\int_{0}^{L} T Y_{n}^{\prime \prime}(z) Y_{n}(z) d z ;$

$P_{n} \quad$ is the modal force and is given by

$P_{n}(t)=\int_{0}^{L} Y_{n}(z) P(z, t) d z$. 
The displacement response at any location $z$ to the excitation with frequency $\omega_{r}$ (in complex form) will be

$$
\bar{y}\left(z ; \omega_{r}\right)=\sum_{n=1}^{\infty} \frac{1}{K_{n}} \frac{\overline{P_{n}} Y_{n}(z)}{\left[1-\left(\frac{\omega_{r}}{\omega_{n}}\right)^{2}+j 2 \zeta_{n} \frac{\omega_{r}}{\omega_{n}}\right]}
$$

Where $\overline{P_{n}}=\int_{z_{x}}^{z_{e}} Y_{n}(z) f(z) d z, \quad \omega_{n}$ is the $\mathrm{n}^{\text {th }}$ natural frequency, and $\zeta_{n}$ is the $\mathrm{n}^{\text {th }}$ damping ratio.

$$
\frac{1}{K_{n}} \frac{1}{\left[1-\left(\frac{\omega_{r}}{\omega_{n}}\right)^{2}+j 2 \zeta_{n} \frac{\omega_{r}}{\omega_{n}}\right]} \text { is the frequency response }
$$

function for mode $\mathrm{n}$. The magnitude of the displacement at location $z$ is then given by

$$
y\left(z ; \omega_{r}\right)=\left|\bar{y}\left(z ; \omega_{r}\right)\right| \text {. }
$$

In solving for the response using mode superposition, the only assumption that has been made is that the response is harmonic in time.

$$
P(z, t)=\operatorname{Re}\left\{f(z) e^{j \omega t}\right\}
$$

When $f(z)$ is a real valued function, such as $\cos (k z+\phi)$, the excitation force is:

$$
P(z, t)=\cos (k z+\phi) \cos (\omega t)
$$

which is the standard form for a standing wave excitation.

As mentioned in the previous section, in order to add the traveling wave characteristic to the response, it is necessary to modify the excitation force model and introduce a function which models a traveling wave. Traveling waves in general have the form

$$
\begin{aligned}
& P(z, t)=g(k z-\omega t+\phi) \quad \text { or } \\
& P(z, t)=g(k z+\omega t+\phi)
\end{aligned}
$$

depending on whether the wave is a left to right traveling or a right to left traveling wave respectively. From equation 13, it can be seen that $P(z, t)$ is of the form shown in equation 15 if $f(z)$ is complex i.e. it is of the form

$$
\bar{f}(z)=\cos (k z+\phi) \pm j \sin (k z+\phi)
$$

The introduction of the second term, $j \sin (k z+\phi)$ adds a traveling wave characteristic to the term on the right hand side of equation 13. This modifying term is just the orthogonal function of the original function $f(z)$ multiplied by the complex number $j$.

In the more general case where $f(z)$ is not sinusoidal, it needs to be first represented as a sum of its Fourier components, i.e.

$$
f(z)=\sum a_{n} \cos \left(k_{n} z\right)+b_{n} \sin \left(k_{n} z\right)
$$

This can also be represented as follows:

$$
f(z)=\sum a_{n} \cos \left(k_{n} z\right)+b_{n} \cos \left(k_{n} z-\pi / 2\right)
$$

Where $k_{n}=\left(n \pi z / L_{i n}\right), L_{i n}$ is the length of the excitation region and the Fourier coefficients $a_{n}$ and $b_{n}$ are evaluated in the conventional way as follows:

$$
\begin{aligned}
& a_{n}=\frac{2}{L_{i n}} \int_{0}^{L_{i n}} f(z) \cos \left(k_{n} z\right) d z \\
& b_{n}=\frac{2}{L_{i n}} \int_{0}^{L_{i n}} f(z) \sin \left(k_{n} z\right) d z
\end{aligned}
$$

The traveling wave excitation can then be written as follows:

$$
\begin{aligned}
& f(z)=\sum a_{n} \cos \left(k_{n} z\right) \pm j a_{n} \sin \left(k_{n} z\right)+ \\
& \sum b_{n} \cos \left(k_{n} z-\pi / 2\right) \pm j b_{n} \sin \left(k_{n} z-\pi / 2\right)
\end{aligned}
$$

Figure 6 shows an example of the predicted response for the Gulf Stream test case 20061023205557 using the modified excitation force model, which consists of a combination of standing and traveling wave excitation. The power-in region between $\mathrm{z} / \mathrm{L}=0$ to 0.1 has a standing wave excitation. This region is marked out by the black bar in the plot on the right. The power-in region between $\mathrm{z} / \mathrm{L}=0.1$ to 0.2 has a traveling wave excitation. This region is marked out by the magenta arrow in the plot on the right. The direction of the traveling wave excitation is from the bottom end towards the top end. The response predicted using this combined standing and traveling wave excitation shows good qualitative match with the measured response. 

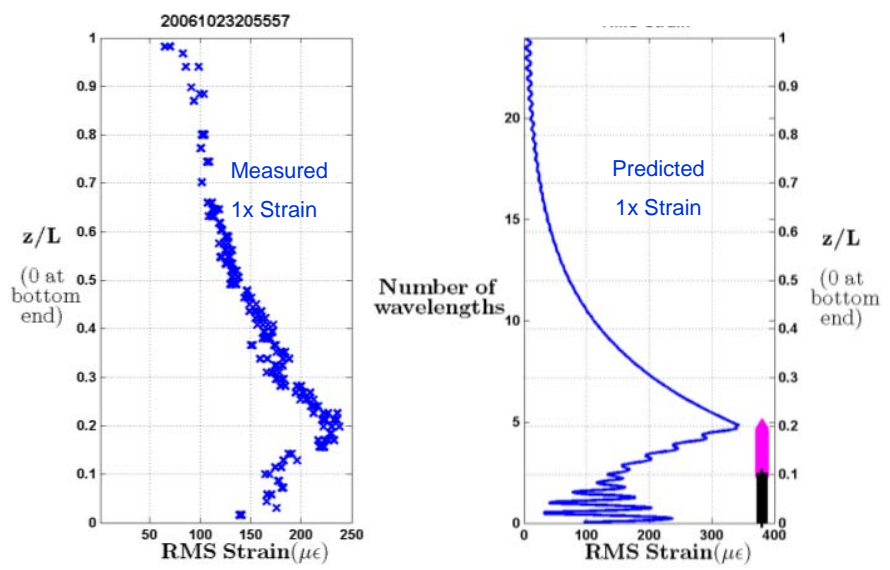

Figure 6 Measured (left) and predicted (right) RMS strain for test case 20061023025557 using the modified excitation model consisting of standing wave excitation between $\mathrm{z} / \mathrm{L}=0$ to 0.1 and traveling wave excitation between $\mathrm{z} / \mathrm{L}=0.1$ to 0.20 .

Figure 7 shows the example of predicted response for the Gulf Stream test case 20061022153003 using the conventional excitation force model. In this example, the power-in region is away from the boundary and the predicted response using the conventional standing wave excitation shows good qualitative match with the measured response.

Figure 8 shows an example of the predicted response for the Gulf Stream test case 20061022153003 using the modified excitation force model, which consists of a combination of standing and traveling wave excitation. The power-in region between $\mathrm{z} / \mathrm{L}=0.18$ to 0.39 has standing wave excitation. This region is marked out by the black bar in the plot on the right. The power-in region between $\mathrm{z} / \mathrm{L}=0.39$ to 0.42 has traveling wave excitation. This region is marked out by the magenta arrow in the plot on the right. The direction of the traveling wave excitation is from the bottom end towards the top end. The inclusion of traveling wave excitation in this case gives the predicted response a sharper peak near $\mathrm{z} / \mathrm{L}=0.42$ and improves the qualitative match between the measured and predicted response.

It is evident from the examples shown above that modifying the excitation force model leads to better qualitative match between the predicted and measured response. Similar improvements in predicted response were obtained for 2 other Gulf Stream test cases where the power-in region was located close to one end of the riser model.

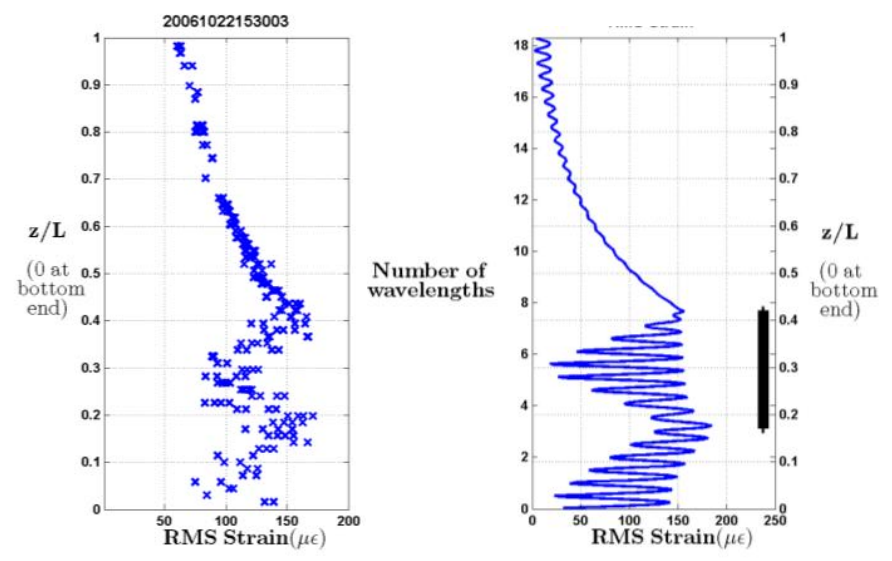

Figure 7 Measured (left) and predicted (right) RMS strain for test case 20061022153003 using the conventional standing wave excitation between $\mathrm{z} / \mathrm{L}=$ 0.18 to 0.42 .

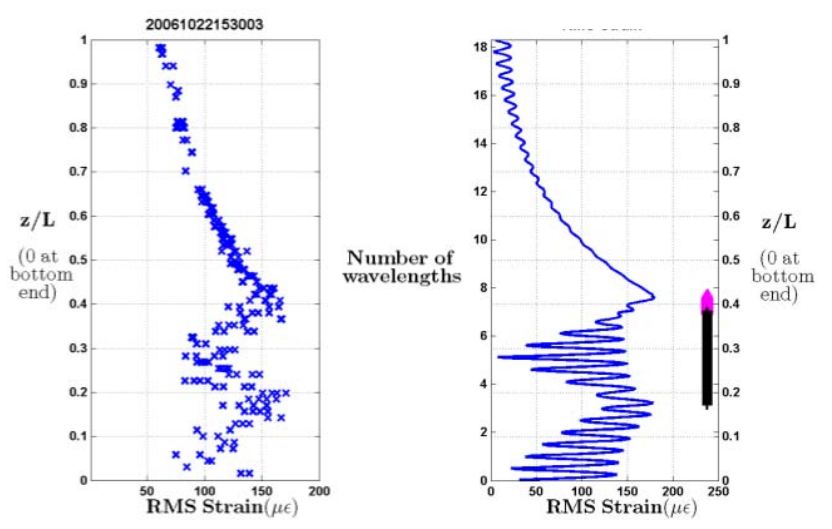

Figure 8 Measured (left) and predicted (right) RMS strain for test case 20061022153003 using the modified excitation model consisting of standing wave excitation between $\mathrm{z} / \mathrm{L}=0.18$ to 0.39 and traveling wave excitation between $\mathrm{z} / \mathrm{L}=0.39$ to 0.42 .

\section{CONCLUSIONS AND DISCUSSION}

It is demonstrated that the VIV response of long flexible cylinders, which is often in the form of traveling waves, can be predicted more accurately using an excitation force model which has traveling wave characteristics. A suitable combination of standing and traveling wave models for the excitation force used in mode superposition predicts a response which is close to the actual data obtained from experiments. The work presented in this paper shows the method for implementing a traveling wave excitation force using mode superposition technique.

The proportions for the standing and traveling wave excitation regions are not well understood at this time. For 
this study, the proportions were obtained from a Green's function based response prediction program. In the Green's function approach, the response at each location along the riser model is the sum of waves traveling in opposite directions. At each location within the power-in region, it is possible to compare the amplitudes of the waves traveling in opposite direction. At a location where the amplitude of the wave traveling in one direction is twice as large as the amplitude of the wave traveling in opposite direction, that location is assumed to have a traveling wave excitation. The direction of the traveling wave excitation is assumed to be the same as the direction of the traveling wave whose amplitude is larger at the location. It is not possible to implement this approach of comparing amplitudes of opposite traveling waves with the mode superposition method; more work is needed to develop the method for determining the regions with traveling wave excitation for the mode superposition method.

\section{ACKNOWLEDGEMENTS}

This research was sponsored by the DEEPSTAR Consortium, the Office of Naval Research Ocean Engineering and Marine Systems program (ONR 321OE) and the SHEAR7 JIP.

\section{REFERENCES}

[1] Jaiswal, V., "Effect of Traveling Waves on VortexInduced Vibration of Long Flexible Cylinders", $\mathrm{PhD}$ thesis, Department of Ocean Engineering, Massachusetts Institute of Technology, Cambridge, USA.

[2] Jaiswal, V. and Vandiver J. K., "VIV Response Prediction for Long Risers with Variable Damping", OMAE2007-29353, 26th International Conference on Offshore Mechanics and Engineering, June 2007, San Diego, California, USA.

[3] Marcollo, H., Chaurasia, H., and Vandiver, J. K., "Phenomena observed in VIV bare riser field tests", 26th International Conference on Offshore Mechanics and Engineering, June 2007, San Diego, California, USA.

[4] Vandiver J. K., Jaiswal, V. And Jhingran V., "Insights on vortex-induced, traveling waves on long risers", Journal of Fluids and Structures 25 (2009) pp 641653.

[5] VIV Data Repository data download page http://oe.mit.edu/VIV/downloadpage.html accessed on 1st of February 2010.

[6] Vandiver, J.K., Leverette, S., Wajnikonis, C.J., Marcollo, H. (2007). "User Guide for SHEAR7 Version 4.5." MIT. Cambridge, Massachusetts, USA 